ORIGINAL ARTICLE

\title{
SEVERITY OF OUTCOMES ASSOCIATED TO ILLNESSES FUNDED BY GFATM INITIATIVE AND SOCIO DEMOGRAPHIC AND ECONOMIC FACTORS ASSOCIATED WITH HIV/AIDS, TB AND MALARIA MORTALITY IN KINSHASA HOSPITALS, DRC
}

\author{
Jose Gaby Tshikuka ${ }^{1,2}$, Léon Okenge $^{2}$, Albert Lukuka ${ }^{3}$, Bibi Mengema ${ }^{2}$, \\ Jacqueline Mafuta $^{2}$, Jerry Itetya ${ }^{2}$, Kimole Ne-Kimole ${ }^{1}$, Gérard Eloko ${ }^{2,4}$
}

ABSTRACT

BACKGROUND: For the past decades, developing countries have received considerable support to fight infectious illnesses in their homelands. This global effort has tremendously reduced case fatality rates associated with illnesses such as HIVIAIDS, tuberculosis and malaria in many countries. However, this information is still missing in some developing countries, hindering international effort for control programs; we designed this study in effort to close this gap.

METHODS: Data on 23,487 inpatients from Kinshasa hospitals were gathered and analyzed using EpiData and SPSS. Major illnesses affecting inpatients were identified; mortality and case fatality rates associated with each such illness were estimated. Case fatality rates associated with each illness were compared between consecutive years. Socio demographic and economic factors associated with mortality due to HIVIAIDS, TB and malaria were investigated using logistic regression.

RESULTS: The outstanding findings were that case fatality rates associated with major illnesses were relatively higher in 2008 than in the previous year; inpatients hospitalized for HIVIAIDS, TB and malaria in 2008 were more likely to die than those hospitalized in the previous year. Low socioeconomic status inpatients hospitalized for malaria, HIV/AIDS or TB were more likely to die than high socioeconomic status inpatients (AOR 0.29, 95\% CI 0.22-0.40; AOR 0.20, 95\%CI0.12-0.33; AOR 0.33, 95\%CI 0.21-0.53), even though both groups presumably had access to free life-saving treatment and care.

CONCLUSION: These results indicate that while improvement in health indicators greatly depends on funds availability and sustainability, these alone might not be enough in resource poor developing countries. Other factors, i.e., population SES also need to be addressed before needed changes may occur.

KEYWORDS: Case fatality rates, Mortality, Socioeconomic status, Funding policies

DOI: http://dx.doi.org/10.4314/ejhs.v24i4.4

\section{INTRODUCTION}

The burden of infectious diseases has been continuously ruthless on developing country populations $(1,2)$, probably due to meagre government spending in the health sectors $(3,4)$ and the lack of expertise and data for planning and formulating effective health Policies (5).

\footnotetext{
${ }^{1}$ Department of Health Science, National Pedagogic University, Kinshasa

${ }^{2}$ Department of Community Health, University School of Medical Technology (ISTM), Kinshasa

${ }^{3}$ National Biomedical Research Institute, (I.N.R.B), Kinshasa

${ }^{4}$ National Control Program for Health Expenditures, General Secretariat, Ministry of Public Health, Kinshasa

Corresponding Author: Jose Gaby Tshikuka Mulumba, Email: jtshikuka@ hotmail.com
} 
Today, however, these claims are no longer enough to justify high mortality and case fatality rates (CFR) related to infectious diseases, particularly to HIV/AIDS, TB and malaria in developing countries $(2,6,7,8)$. This is because for the past decades, these countries receive, through international partnership, considerable and sustainable support to fight major infectious diseases in their homelands (8). The Global Fund to Fight AIDS, TB and Malaria (GFATM) for instance, has been attracting and disbursing additional funds to prevent and treat HIV/AIDS, tuberculosis and malaria in endemic regions for many years. From its launching in 2002 by the G8 to 2010, GFATM had committed more than US $\$ 22$ billion in 150 countries $(9,10)$. Allegedly, every population in these countries has access not only to free life-saving antiretroviral treatment (ART) and TB drugs and care, but also to largescale prevention programs against the three pandemics (9). The US Center for Diseases Control (CDC), through its global infectious diseases strategies, has been very active in endemic areas for many years, bringing in experts, logistics and funds to fight infectious diseases (11, 12). Adding to that is the implication of organizations such as the Canadian International Development Agency (CIDA), UNICEF and Population Services International (PSI), to name but a few, in strengthening health sectors in developing countries (13).

This international effort has brought tremendous improvement on health indicators in many developing countries, particularly on HIV, TB and malaria $(11,14,15)$. In his address at the $22^{\text {nd }}$ board meeting in Sofia, the Global Fund's Executive Director, made it clear that the scaling up of malaria interventions was reducing childhood mortality, and that the number of new cases of HIV/AIDS had fallen globally by more than $20 \%$ since 1997 . TB-related mortality went down by more than a third from 1990 (9). While these statements bring new hope to our battle against infectious diseases, data from Democratic Republic of Congo (DRC) are indicating that the majority of deaths in this country are still due to infectious diseases (16), particularly malaria, HIV/AIDS and TB; seemingly because of severe poverty among populations and limited access to health care facilities (17). Reportedly (18), populations with lower socioeconomic status
(LSES) have limited access to free malaria protection tools than those with higher socioeconomic status (HSES), thus resulting in higher childhood mortality among rural poor populations compared to urban dwellers $(13,16)$. While these data are of great relevance for policy making, they would have been of even more interest had they highlighted case fatality rates (CFRs) of illnesses such as HIV/AIDS, TB and malaria. Since, every population in the country allegedly has access to free life-saving treatment and care for these illnesses. Such data would have not only highlighted any decline on the severity of outcome associated with AIDS, TB and malaria, but would have also assessed the effectiveness of control and prevention measures targeting predictors of mortality related to these diseases $(18,16)$.

In 1989, Greenberg and coworkers estimated at $21 \%$ the severity of outcome associated with malaria in the city of Kinshasa (6), whether or not this rate has declined is still unknown. Though works by Tshikuka and collaborators (17) in this same city estimated CFRs of HIV co-infections with TB and malaria, CFRs associated with each specific illness and/or major causes of hospital admission were not examined. This study collected and analyzed data from hospitals in Kinshasa to address not only the missing information, but also factors associated with HIV/AIDS, TB and malaria mortality so as to provide stakeholders with enough evidence to tailor new policies and implementation strategies to address the burden of HIV/AIDS, TB, malaria and other infectious illnesses in developing countries. Our objectives were to: (i) identify major causes of hospital admissions in Kinshasa, DRC; (ii) estimate the severity of outcome or CFR associated with each such illness; (iii) compare CFRs associated to each illness between consecutive years; and (iv) to examine associations of mortality related to HIV/AIDS, TB and malaria with inpatients' socio demographic and economic characteristics.

\section{SUBJECT AND METHODS}

The study was conducted in 14 hospitals in Kinshasa, DRC from 2007 to 2008 using a cross sectional retrospective survey and quantitative approach. At the time when the study was 
conducted, the city had 66 hospitals. Admission fees of up to US $\$ 1,000$ or more were charged by some hospitals, i.e., at Kinshasa Medical Center. Doctors often waited for inpatients or their relatives to bring money, medicines or even basic medical supplies such as bandages, before initiating treatment (16). Those who could not afford doctors' requests received no treatment while in hospitals (16). Some hospitals had private wards where inpatients paid extra money to receive special care. Twenty three of the 66 hospitals were selected at random; after a checkup of the quality of their record keeping, 9 were dropped and 14 were kept in the study.

Hospital admission books, patient record files, hospital discharge books and mortuary record books were matched and data collected for 2007 and 2008. From each inpatient, 21 data items were collected. They included the following: patient's name, date of birth, gender, occupation, neighborhood of residence, municipality of residence, date of illness onset, admission date, the main causes of hospitalization (diagnosis 1,2 and 3 ) coded as by the international classification of diseases, ICD -10 ; whether or not patients stayed in a private ward, whether or not patient's relatives brought money, medicine or medical supply requested by the doctor; admission fees paid, year patient was admitted, year patient was discharged from the hospital, whether patient was cured or dead upon discharge, whether patient was transferred to another facility; whether he/she escaped from hospital, name of the hospital, number of hospital beds, number of nurses, number of doctors. We recorded patients who afforded private wards and/or paid admission fees of US\$1,000 or more as having a higher SES (20). Patients admitted twice or more times with the same condition within the same year were counted as single case for that year. Apart from data obtained from hospital records, no contact was made with respondents or surrogates to obtain additional information on patients. Information on the severity of illnesses at admission was missing from record books; hence, patients who died or were discharged after less than 24 hours of admission, as well as bodies brought to mortuaries simply because of death certificates were excluded from the analysis. Also, excluded from the study were patients hospitalized for reasons other than medical condition, i.e., birth.
Double data entry was performed and assessment done using EpiData 3.1 (CDC, Atlanta, Georgia, USA); SPSS 16 (Gorinchem, Netherlands) in data analysis. The frequency distribution (\%) of inpatients by cause of hospitalization was assessed for 2007 and 2008. CFR (\%) of every illness was estimated as the ratio of the number of deaths associated with a particular illness over the number of inpatients admitted for that specific illness, times 100 . We compared CFRs between 2007 and 2008 using Chi-Square analysis with Yates' Correction. Associations of malaria, HIV/AIDS and TBrelated deaths with socio demographic and economic characteristics were investigated using three logistic regression sub-models (21): (i) submodel A with malaria-related deaths as dependent variable, (ii) sub-model B with HIV/AIDS-related deaths as dependent variable and, (iii) sub-model $\mathrm{C}$ with TB-related deaths as dependent variable. The following variables were investigated as potential predictors of mortality to compute for adjusted odds ratios (AOR) and their 95\% confidence intervals as approximations of adjusted relative risks: inpatient SES, age, gender, area of residence, municipality of residence, month of illness onset and year of hospitalization. Numbers of hospital beds per doctor, the number of hospital beds per nurse and malnutrition among inpatients were investigated as potential confounders. As HIV concurrent infections were already discussed elsewhere (17), HIV inpatients co-infected with TB and/or malaria, were all included in the analysis as HIV/AIDS patients only. Redundancy and multicollinearity of covariates were checked using the tolerance/VIF diagnostic. All variables significant in the main effect models (as well as those suspected to have different relationships with the outcome variable depending on the third factor) were investigated in a series of interaction term. How well the models fit the data were estimated using the Hosmer-Lemeshow test of goodness of fit. The level of significance was set at $\mathrm{P}<0.05$.

The School of Public Health Ethical Committees provides general oversight and ethical approval in DRC. This committee does not require a formal submission for ethical approval for the type of study presented herein. However, consent from hospital managements was obtained by confirming 
that the data will be used only for research purpose and no contact be made with patients.

\section{RESULTS}

Selected hospitals had a total capacity of 4,796 beds and healthcare services were provided by 922 doctors and 3,175 nurses. There were no records of ART, TB or malaria drug supply breakdowns in selected hospitals throughout the study period. Diagnostic tests used in all these hospitals were similar and met the national standard.

Data came from 23,487 inpatients: 13,358 (57\%) from 2007 records and 10,129 (43\%) from 2008 records; $11,558(49.2 \%)$ patients were male and $11,929(50.8 \%)$ were female. The median age was 21 years (interquartile range: 3 - 37 years). The majority of inpatients, 18,806 (80.1\%), recovered from their illnesses upon discharge, yet $2,421(10.3 \%)$ died in hospitals; 1,807 (7.7\%) patients were transferred from one hospital to other facilities, while 453 (1.9\%) near recovery patients escaped from hospitals before discharge. Only $19.5 \%$ of inpatients were considered as having HSES, while the rest, $80.5 \%$, were categorized as having LSES. Data also showed that $49.2 \%$ inpatients lived in semi-urban areas and $50.8 \%$ lived in urban areas; only $10.9 \%$ of inpatients lived in rich neighborhoods.

Data presented in Table 1 show the distribution pattern of inpatients by cause of hospitalization in 2007 and 2008: $37.4 \%$ of admissions were due to malaria alone during the two-year study period; $7.5 \%$ were due to acute respiratory infection (ARI), 5.8\% to diarrhea, $5.6 \%$ to TB, $5.5 \%$ to HIV/AIDS, $2.6 \%$ to traffic injuries (TI), $2.5 \%$ to malignancies, $2.2 \%$ to malnutrition, $1.7 \%$ to sexually transmitted diseases (STD), $1.4 \%$ to vaccine preventable diseases (VPD) and $27.7 \%$ of hospitalizations were due to other illnesses.

Table 1: Distribution pattern (\%) of inpatients by cause of hospitalization in 2007 and 2008 in 14 Kinshasa Hospitals, DRC

\begin{tabular}{lllllll}
\hline Cause of Hospitalization & \multicolumn{3}{l}{ Year of Hospitalization } & \multicolumn{3}{c}{ Total } \\
\cline { 2 - 5 } & $\mathbf{2 0 0 7}$ & \multicolumn{2008}{c}{} & & & \\
Malaria & Case & \% & Case & \% & Case & \% \\
ARI & 4916 & 36.8 & 3858 & 38.1 & 8774 & 37.4 \\
Diarrhea & 905 & 6.8 & 867 & 8.6 & 1772 & 7.5 \\
TB & 679 & 5.1 & 688 & 6.8 & 1367 & 5.8 \\
HIV/AIDS & 642 & 4.8 & 683 & 6.7 & 1325 & 5.6 \\
TI & 651 & 4.9 & 651 & 6.4 & 1302 & 5.5 \\
Malignancies & 308 & 2.3 & 295 & 2.9 & 603 & 2.6 \\
Malnutrition & 339 & 2.5 & 253 & 2.5 & 592 & 2.5 \\
STD & 216 & 1.6 & 309 & 3.1 & 525 & 2.2 \\
VPD & 207 & 1.5 & 183 & 1.8 & 390 & 1.7 \\
Others & 169 & 1.3 & 164 & 1.6 & 333 & 1.4 \\
\hline All Causes & 4326 & 32.4 & 2178 & 21.5 & 6504 & 27.7 \\
\hline Legend: ARI & 13358 & 100 & 10129 & 100 & 23487 & 100 \\
\hline
\end{tabular}

Legend: $\mathrm{ARI}=$ acute respiratory infections; TB =Tuberculosis; HIV =human immunodeficiency virus; AIDS =acquired immunodeficiency syndrome; TI=traffic injuries; STD =sexually transmitted diseases; VPD $=$ vaccine preventable diseases; others $=$ all other diseases

CFRs associated to each cause of admission are presented in Table 2. Significant $(\mathrm{P}<0.05)$ increases are observed in malaria, HIV/AIDS, ARI, VPD and TB $(\mathrm{P}=0.06)$ in 2008 compared to
2007. Of all causes of hospitalization, malignancies have the highest CFR, while malaria has the lowest. 
Table 2: Severity of outcomes or case fatality rates (CFR \%) associated to major causes of hospitalization among 23,487 inpatients, Kinshasa, DRC in 2007 and 2008

\begin{tabular}{|c|c|c|c|c|c|c|}
\hline \multirow{3}{*}{ Condition } & \multicolumn{4}{|c|}{${ }^{\circledR}$ Year of Hospitalization } & \multirow{2}{*}{\multicolumn{2}{|c|}{ Total }} \\
\hline & 2007 & & 2008 & & & \\
\hline & Death/case & CFR \% & Death/case & CFR\% & Death/case & CFR\% \\
\hline Malaria* & $256 / 4916$ & $5.2^{\mathrm{a}}$ & $319 / 3858$ & $8.3^{\mathrm{b}}$ & $575 / 8774$ & 6.6 \\
\hline HIV/AIDS* & $126 / 651$ & $19.4^{\mathrm{a}}$ & 165651 & $25.3^{\mathrm{b}}$ & $291 / 1302$ & 22.4 \\
\hline $\mathrm{ARI}^{*}$ & $98 / 905$ & $10.8^{\mathrm{a}}$ & $186 / 867$ & $21.5^{\mathrm{b}}$ & $284 / 1772$ & 16.0 \\
\hline Diarrhea & $119 / 679$ & 17.5 & $144 / 688$ & 20.9 & $263 / 1367$ & 19. 2 \\
\hline $\mathrm{TB}^{\psi}$ & $109 / 642$ & $17.0^{\mathrm{a}}$ & $143 / 683$ & $20.9^{\mathrm{b}}$ & $252 / 1325$ & 19.0 \\
\hline Malignancy & $110 / 339$ & 32.4 & $74 / 253$ & 29.2 & $184 / 592$ & 31.1 \\
\hline STD & $41 / 207$ & 19.8 & $35 / 183$ & 19.1 & 76/390 & 19.5 \\
\hline TI & $31 / 308$ & 10.1 & $31 / 295$ & 10.5 & $62 / 603$ & 10.3 \\
\hline Malnutrition & $28 / 216$ & 13.0 & $32 / 309$ & 10.4 & $60 / 525$ & 11.4 \\
\hline VPD* & $15 / 169$ & $8.9^{\mathrm{a}}$ & $29 / 164$ & $17.7^{\mathrm{b}}$ & $44 / 333$ & 13.2 \\
\hline Others* & $251 / 4326$ & $5.8^{\mathrm{a}}$ & $79 / 2178$ & $3.6^{\mathrm{b}}$ & $330 / 6504$ & 5.1 \\
\hline All Conditions* & $1184 / 13348$ & $8.9^{\mathrm{a}}$ & $1237 / 10129$ & $12.2^{b}$ & 2421/23487 & 10.3 \\
\hline
\end{tabular}

Legend: $* \mathrm{P}<0.05 ;{ }^{*}$ borderline significance $\mathrm{P}=0.06$; ARI $=$ acute respiratory infections; $\mathrm{TB}=$ Tuberculosis; $\mathrm{HIV}=$ human immunodeficiency virus; AIDS = acquired immunodeficiency syndrome; TI = traffic injuries; STD = sexually transmitted diseases; VPD = vaccine preventable diseases; others = all other conditions; ${ }^{\circledR}$ different superscript letters within a row indicate significant difference $(\mathrm{P}<0.05)$

Multivariate sub-models presented in Table 3 reveal that patients hospitalized for malaria, HIV, and TB in 2008 were more likely to die from these diseases than patients hospitalized in 2007 with the same conditions. Results also indicate that LSES inpatients admitted for malaria, TB and HIV/AIDS were more likely to die than HSES inpatients admitted for the same diseases; resident of semi-urban areas were more likely to die from malaria than those from urban areas. Only HIV/AIDS or TB inpatients aged $0-13$ years old were less likely to die from these conditions compared to inpatients aged $\geq 50$ years old. Inpatients aged $14-49$ years old had the same risk of dying of $\mathrm{HIV}(\mathrm{P}=0.44)$ or TB $(\mathrm{P}=0.36)$ as inpatients aged $\geq 50$ years old.

\section{DISCUSSION}

Outstanding findings in this study were that severities of outcomes associated with major illnesses in 2008 were relatively higher than in the previous year. In 2008 for instance, malaria CFR was $8.3 \%$ compared to $5.2 \%$ in 2007 ; HIV CFR was $25.3 \%$ compared to $19.4 \%$ in 2007 ; TB CFR was $20.9 \%$ compared to $17 \%$ in 2007 . This clearly suggests that the quality of care provided to patients and control and prevention measures implemented in 2008 were less effective than in 2007 (22). Yet, looking at resources allocated to the three diseases by the GFATM initiative alone (9), there was potential for decrease in CFRs of these illnesses each consecutive year (22). Our present study points to the contrary. Indeed, reports from Kinshasa Provincial Health Authority (19) also indicated substantial increase in malaria related mortality in 2008 than the previous years, thus lending support to our results, and at the same time, raising questions on factors responsible for such an increase.

Our multivariate analysis to highlight factors associated with such mortality also revealed that inpatients hospitalized for HIV/AIDS, TB and malaria in 2008 were more likely to die than those hospitalized in 2007 for the same diseases; and that LSES inpatients admitted for malaria, TB or HIV/AIDS were more likely to die than HSES inpatients admitted for the same diseases. While on one hand these results lead to speculations that between 2007 and 2008 no progress was made in this city toward meeting GFATM objectives, they also suggest that in resource poor settings, the likelihood of dying from malaria, HIV/AIDS or TB is still associated with poverty (23), despite presumably free access to life-saving treatment and care for these diseases (10). 
Table 3: Socio-demographic and economic factors independently associated with malaria, HIV/AIDS, and TB-related deaths among inpatients in Hospitals in 2007 and 2008.

Sub Model A

Dependent Variable: Malaria-related Death $(\mathbf{N}=\mathbf{8 , 7 7 4})$

\begin{tabular}{llllll}
\hline & Proportion\% & Unadjusted & \multicolumn{3}{c}{ Adjusted } \\
\hline Independent Variables & & OR & $95 \%$ CI & OR & 95\%CI \\
Year: 2007 & 44.5 & 1 & & 1 & \\
2008 & 55.5 & $1.64^{*}$ & $1.38-1.95$ & $1.85^{*}$ & $1.55-2.19$ \\
SES: Low & 90.8 & 1 & & 1 & \\
$\quad$ High & 9.2 & $0.47^{*}$ & $0.36-0.63$ & $0.29 *$ & $0.22-0.40$ \\
AR: Semi urban & 54.4 & 1 & & 1 & \\
$\quad$ Urban & 45.6 & $0.67^{*}$ & $0.56-0.80$ & $0.49 *$ & $0.45-0.58$ \\
\hline
\end{tabular}

Hosmer-Lemeshow test of goodness of fit $\psi \mathrm{P}=0.05$

Sub Model B

Dependent Variable: HIV/AIDS-related Death $(\mathrm{N}=1,302)$

\begin{tabular}{clllll}
\hline & Proportion\% & Unadjusted & \multicolumn{2}{l}{ Adjusted } \\
\hline Independent Variables & & OR & $95 \% \mathrm{CI}$ & OR & $95 \% \mathrm{CI}$ \\
Year: 2007 & 43.3 & 1 & & 1 & \\
2008 & 56.7 & $1.42^{*}$ & $1.1-1.8$ & $2.15^{*}$ & $1.61-2.85$ \\
SES: Low & 93.0 & 1 & & 1 & \\
High & 7.0 & $0.29^{*}$ & $0.18-0.46$ & $0.20^{*}$ & $0.12-0.33$ \\
Age group: $\geq 50$ years & 27.5 & 1 & & 1 & \\
$0-13$ years & 9.6 & $0.23^{*}$ & $0.15-0.37$ & $0.18^{*}$ & $0.11-0.30$ \\
$14-49$ years & 62.9 & 1.07 & $0.78-1.46$ & 1.14 & $0.83-1.56$ \\
\hline
\end{tabular}

Hosmer-Lemeshow test of goodness of fit $\mathrm{P}=0.41$

\section{Sub Model C}

Dependent Variable: TB-related Death $(\mathbf{N}=1,325)$

\begin{tabular}{clllll}
\hline & Proportion\% & Unadjusted & \multicolumn{2}{l}{ Adjusted } \\
\hline Independent Variables & & OR & 95\%CI & OR & $95 \% \mathrm{CI}$ \\
Year: 2007 & 43.3 & 1 & & 1 & \\
2008 & 56.7 & 1.29 & $0.98-1.7$ & $1.32^{\psi}$ & $0.99-1.75$ \\
SES: Low & 91.3 & 1 & & 1 & \\
High & 8.7 & 0.41 & $0.26-0.6)$ & $0.33^{*}$ & $0.21-0.53$ \\
Age group: $\geq 50$ years & 23.0 & 1 & & 1 & \\
0 - 13 years & 9.1 & $0.23^{*}$ & $0.14-0.38$ & $0.21^{*}$ & $0.12-0.35$ \\
14-49 years & 67.9 & 0.87 & $0.62-1.20$ & 0.85 & $0.60-1.20$ \\
\hline
\end{tabular}

Hosmer-Lemeshow test of goodness of fit $\mathrm{P}=0.29$

Legend: Year $=$ year of hospitalization; SES $=$ socioeconomic status; age groups $=(0-13$ years old, $14-49$ years old, $\geq 50$ years old; reference group $\geq 50$ years old); $\mathrm{AR}=$ area of residence (semi-urban area, urban area); $* \mathrm{P}<0.05$, "borderline significance $(\mathrm{P}=0.05)$

These findings are strongly supported by others $(24,25)$, even though they do not explain why LSES patients are more at risk of dying than HSES patients, seeing that both groups presumably had access to free life-saving treatment and care for TB and HIV/AIDS. If Wood et al (25) found LSES patients more at risk of dying from HIV/AIDS than HSES patients, it was quite normal because in their study, LSES patients were less likely to be prescribed Highly Active Antiretroviral Treatment (HAART). Where we conducted this study, HIV and TB drugs and care are meant to be totally free (9). Also, treatment of severe malaria is highly subsidized; 
in which case, the difference in CFRs between the two groups cannot be justified.

However, in resource poor settings like Kinshasa, where healthcare professionals struggle for their own survival and often request money, medicines and even basic medical supplies from inpatients or their relatives before initiating treatment (16), it is hard to believe that there were no hurdles for LSES inpatients to access free lifesaving ART or TB drugs and care (16). In addition, the fact that inpatients' food rations had to come from their families outside hospitals, differences in diet might also have had varying impacts on patients' response to treatment (26).

Other key findings in this study were that: inpatients from semi-urban areas were more likely to die from malaria compared to those from urban areas. This lends support to existing data (15). While inpatients aged $0-13$ years were less likely to die from HIV/AIDS and TB compared to $\geq 50$ years old reference group, inpatients aged $14-49$ years had the same risk of dying from HIV/AIDS $(\mathrm{P}=0.44)$ and TB $(\mathrm{P}=0.36)$ as those aged $\geq 50$ years old likely because of uncontrolled underlying medical conditions (27).

Our non-inclusion of underlying medical conditions, i.e., diabetes mellitus, and renal failure (27), in the analysis as potential confounders might be a limiting factor. Other limiting factors might come from the use of hospital data to study mortality (28) and case fatality in a developing country setting where not every sick person attends hospitals, and burials often occur without the knowledge of the cause of death which might have underestimated outcomes. Hospital data in developing countries are often of poor quality and differences among groups may come from variables other than those under study, i.e., epidemics, improvement in recording system, etc. Nevertheless, data that are regularly generated in hospitals have been effectively used for epidemiological purposes $(6,27)$ as they are inexpensive and readily available. And, in developing countries like DRC where health data are often lacking, hospital data can provide good evidence for planning and decision making if they are properly collected and processed (6). In this work, we meticulously addressed these issues; of the 23 hospitals that were preliminary selected, 9 were dropped for poor record keeping. Patients whose data were missing or could not be matched between different sources within the same hospital were excluded from the study. Based on this, we are doubtful that factors other than those discussed here explain the results.

In short, findings in this study indicate that while the impact of health programs on indicators greatly depends on availability and sustainability of funds, these alone might not be enough in resource poor developing countries. Other factors, i.e., population SES also need to be addressed before needed changes may occur. It is hoped that this discussion will stimulate research for more evidence on socioeconomic factors association with population health indicators as a base for policy and decision making in a global effort to tailor new approaches to address the burden of diseases in developing countries.

\section{ACKNOWLEDGMENTS}

We gratefully acknowledge the technical support received from the National Malaria Control Program, $(P N L P)$. We express our gratitude to $\mathrm{Mr}$. Mulumba Tshikuka Jr., Writer, for excellent editing. Special thank goes to all health professionals in study sites for their cooperation. The study was funded by the student research and dissertation funds at University School of Medical Technology, DRC.

\section{REFERENCES}

1. WHO: Report on Infectious Diseaes, Code: WHO/CDS/99.1, 1999.

2. Mukadi Y D, Mahera D, Harriesb A. Tuberculosis case fatality rates in high HIV prevalence populations in sub-Saharan Africa. AIDS 2001; 15: 143 - 152.

3. World Bank: Democratic Republic of Congo Public Expenditure Review (PER). Department for International Development, 2008.https://openknowledge.worldbank.org/ha ndle/10986/8117

4. Anon ${ }^{1}$ : DRC Government Global Budget and Health Budget from 1998-2009. Tulane University School of Public Health, New Orleans, L.A, 2010. http://familyplanningdrc.net/financing-govern

5. Walt $\mathrm{G}$ and Gilson L. Reforming the health sector in developing countries: the central role 
of policy analysis. Health Policy Plan 1994; 9: 353-370.

6. Greenberg A E, Ntumbanzondo M, Ntula N, et al. Hospital-based surveillance of malariarelated paediatric morbidity and mortality in Kinshasa, Zaire. Bull World Health Organ 1989; 67: 189-196.

7. Churchyard G J, Kleinschmidt I, Corbett E L, et al. Factors Associated with an Increased Case-Fatality Rate in HIV-Infected and NonInfected South African Gold Miners with pulmonary tuberculosis. Int J Tuberc Lung Dis 2000; 4: 705-712

8. Spicer N, and Walsh A. 10 best resources on the current effects of global health initiatives on country health systems. Health Policy Plan 2012; 27: 265-269.

9. Anon ${ }^{2}$ : The Global Fund to Fight AIDS, Tuberculosis and Malaria. The Global Fund Annual Report. 2010, ISBN 978 - 92 - 9224 $270-1$.

10. Kerouedan D. The Global Fund to fight HIV/AIDS, Tuberculosis and Malaria Fiveyear Evaluation Policy Challenges Field $\begin{array}{llll}\text { Actions } & \text { Sc } & \text { Rep } & 2010 .\end{array}$ http://factsreports.revues.org/635

11. WHO: The world health report: working together for health, Geneva, Switzerland, 2006. http://www.who.int/whr/2006/en

12. CDC: CDC's Vision for Public Health Surveillance in the $21^{\text {st }}$ Century MMWR; 2012, 61.

13. Costello A, White H. Reducing global inequalities in child health. Arch Dis Child 2001; 84: 98-102.

14. Zhang F, Dou Z, Ma Y, et al. Five-year outcomes of the China National Free Antiretroviral Treatment Program. Ann Intern Med 2009; 151: 241 - 215.

15. Jima D, Getachew A, Bilak H, et al. Malaria indicator survey 2007, Ethiopia: coverage and use of major malaria prevention and control interventions. Malar $J \quad 2010$. http://www.malariajournal.com/content/9/1/58

16. Coghlan B, Brennan R J, Ngoy P, et al. Mortality in the Democratic Republic of Congo: a nationwide survey. Lancet 2006; 367: 44-51.
17. Tshikuka J G, Atua B M, Lukuka A K, et al. Severity of Outcomes Associated to Types of HIV Coinfection with TB and Malaria by SES in a Setting where the Three Pandemics Overlap. J Community Health 2012; 37: 12341238.

18. DHS: Democratic Republic of Congo. Demographic and Health Survey, Ministry of Planning 4155, rue des Coteaux Quartier Petit Pont Kinshasa/Gombe, DRC, 2007.

19. Anon $^{3}$ : Rapport Annuel d'Activités, Inspection Provinciale de la Santé, Ministère de la Santé, Kinshasa/Gombe, DRC, 2009.

20. Susser M W, Watson W, Hopper K. Sociology in Medicine. New York, NY: Oxford University Press, 1985.

21. Schlesselman J J. Case-Control Studies. Design, Conduct, Analysis. Oxford University Press: Oxford, 1982.

22. Thai S, Koole O, Un P. Five-year experience with scaling-up access to antiretroviral treatment in an HIV care programme in Cambodia. Trop Med Intern Health 2009; 14: 1048-1058.

23. Syme S L, Berkman L F. Social Class, Susceptibility and sickness. Am J Epidemiol 1976; 104: 1 - 8.

24. Marmot M G, Shipley M J, Rose G. Inequalities in death - specific explanations of a general pattern. Lancet 1984; 1:1003-1006.

25. Wood E, Montaner J S G, Chan K, et al. Socioeconomic status, access to triple therapy, and survival from HIV-disease since 1996. AIDS 2002; 16: 2065-2072.

26. Anon ${ }^{4}$. Position of dietitians of Canada and the American Dietetic Association: nutrition intervention in the care of persons with human immunodeficiency virus infection. Can J Diet Pract Re 2000; 61:77-78.

27. Fielder J F, Chaulk C P, Dalvi M, et al. High Tuberculosis Case-Fatality Rate in a Setting of Effective Tuberculosis control: Implications for Acceptable Treatment Success Rates. Int $J$ Tuberc Lung Dis 2002; 6: 1114-7A.

28. Shahian D M, Wolf R E, Iezzoni L I. Variability in the Measurement of Hospitalwide Mortality Rates. N Engl J Med 2010; 363: 2530-2539. 